\title{
La formación continua en el sector financiero: implicaciones para las mujeres de más de 45 años
}

\author{
Esther Fernández Mostaza \\ Universidad Autónoma de Barcelona \\ Isabel Vidal \\ Universidad de Barcelona
}

RESUMEN

\begin{abstract}
El objeto de estudio es la descripción de las actuaciones en formación continua que actualmente operan en el sector de la intermediación financiera, y más concretamente en el colectivo de mujeres de entre 45 y 55 años, empleadas en el sector bancario durante más de veinte años dentro de categorías poco cualificadas. Con la intención de poner de manifiesto que los determinantes de quién recibe formación en la empresa están muy relacionados con los costes y rendimiento de la inversión en capital humano, dos grandes cuestiones vertebran el núcleo fundamental del artículo: en primer lugar, ¿qué características definen y diferencian la generación de mujeres a la que nos referimos? Una vez trazadas las características, la cuestión es: ¿se benefician estas mujeres de los programas de formación continua de las empresas? Y si no es así, ¿por qué?
\end{abstract}

Palabras clave: Formación continua, Tecnología de la Información, Sector financiero, Mujeres trabajadoras. 


\section{INTRODUCCIÓN}

Si pensamos en aquellos sectores que en los últimos veinte años hayan protagonizado profundas y rápidas transformaciones, coincidiremos en afirmar que el sector financiero y bancario sería un buen ejemplo. Concretamente, en el caso del sector bancario español, la combinación de dos grandes fuerzas ha acelerado los cambios (Martín Fernandez, 2000): la liberalización y desregulación de los mercados financieros y la innovación tecnológica en los campos de la información. El presente artículo se enmarca justamente en la línea de investigación que destaca los cambios que las Tecnologías de la Información y la Comunicación (TICs) han impuesto al sector de intermediación financiera, prestando especial atención al tipo de formación continua que su incorporación exige.

Nuestro objeto de estudio se centra en la descripción de las actuaciones en formación continua que actualmente operan en el sector de la intermediación financiera. Si bien se han elaborado numerosos estudios sobre el tema de la formación continua, en el trabajo que presentamos nos hemos interesado, de entre los empleados que potencialmente se pueden beneficiar de las cualificaciones adicionales proporcionadas por la empresa, en el colectivo de mujeres mayores de 45 años, empleadas en el sector bancario durante más de veinte años. La gran mayoría de estas mujeres ha vivido varias fusiones y compras de sus respectivas entidades bancarias y en la actualidad, en gran parte debido a la incorporación de las TICs, la actividad meramente administrativa que desarrollaban se ha desplazado hacia la venta de productos financieros.

El presente artículo tiene como objetivo poner de manifiesto que los determinantes de quién recibe formación en la empresa están muy relacionados con los costes y rendimiento de la inversión en capital humano (Peraita, 2000). El proceso de selección para tomar parte en actividades de formación está condicionado al objetivo de asegurar que los empleados que la reciben proporcionarán los mayores aumentos de productividad para su empresa durante el mayor período de tiempo posible. Por tanto, características como la edad, el género, el nivel de educación, la ocupación y la antigüedad en la empresa están relacionadas con el anterior proceso de selección para determinar quién recibirá qué formación en la empresa.

Así, dos grandes cuestiones vertebrarán el presente artículo. En primer lugar hemos buscado responder a la pregunta: ¿qué características definen y diferencian la generación de mujeres a la que nos referimos? Una vez trazadas estas características nos hemos preguntado: ¿se benefician estas mujeres mayores de 45 años, que llevan más de veinte trabajando en el sector de intermediación financiera, de los programas de formación continua de sus empresas? Y si no es así, ¿por qué razones? 
Siguiendo estas dos grandes cuestiones, el artículo que presentamos se estructura del modo siguiente: comenzaremos con un apartado dedicado a la metodología empleada en este trabajo. En el apartado segundo presentaremos las características de las mujeres que centran el interés del presente estudio; a tal fin, primero, estructuramos su vida laboral en dos períodos: la primera vida adulta (con el acceso al empleo y los años que le siguen) y la segunda vida adulta (con la prejubilación en el horizonte). En el apartado tercero analizaremos la formación continua en la empresa dentro de un mercado laboral regulado como el español, en el marco de la teoría de la formación en mercados no competitivos. Finalmente, presentaremos las conclusiones más importantes a las que hemos llegado.

\section{METODOLOGÍA}

El trabajo que presentamos se enmarca dentro del proyecto Servemploi ${ }^{1}$ : Un estudio sobre el empleo de la mujer y las perspectivas de trabajo en la Sociedad de la Información, en el programa TSER, Targetted Socio-Economic Research, de la Comisión Europea, y que comenzó su tarea investigadora a principios de 1999. El proyecto, de tres años de duración, incluye la participación de ocho países de la Unión Europea y tiene como objetivos principales los siguientes: en primer lugar, examinar los patrones y la dinámica de innovación tecnológica que operan en dos sectores de la Sociedad de la Información: el comercio minorista y el de la intermediación financiera. En segundo lugar, evaluar las oportunidades para que las trabajadoras a las que nos referimos desarrollen y utilicen estos conocimientos dentro del contexto de los patrones de cambio. Finalmente, el tercer objetivo del proyecto es el de comparar y contrastar transnacionalmente estos patrones de cambio y las experiencias que conllevan.

Los investigadores en los ocho países que participan en el estudio - Dinamarca, Suecia, Irlanda, Reino Unido, Francia, Alemania, Italia y España- estudiamos los avances clave en dos sectores en los que crecientes cambios vinculados a los avances en la Sociedad de la Información han sido y son significativos para los respectivos países. (Por la lógica limitación de espacio, en el presente artículo sólo nos referiremos al caso español.) A tal fin, por un lado, los estudios se centran en entidades que ofrecen la venta minorista y los servicios financieros y, por otro, investigamos el trabajo realizado, las técnicas utilizadas y las perspectivas de desarrollo personal que tienen las mujeres empleadas en puestos no directivos en cada uno de estos sectores.

La lógica metodológica que ha guiado el estudio es la cualitativa, no centrándonos en métodos estructurados en la forma de obtener respuestas de las entrevistadas, sino en permi-

\footnotetext{
1 http://www.tcd.ie/ERC/Servemploi/index.htm.
} 
tir que el discurso emergiera y se fuera repitiendo, con matices diferentes, sesión tras sesión. Dentro de la metodología cualitativa hemos hecho uso de los estudios longitudinales y de los estudios de caso. Por estudio longitudinal nos referimos a una serie de entrevistas en profundidad que cada país que participa en el estudio ha realizado a un panel de tres mujeres empleadas en cada uno de los sectores estudiados - el sector financiero y el del comercio minorista-, con una periodicidad bimensual durante dos años y medio. El objetivo que dicha metodología persigue es doble: en primer lugar, mostrar en qué medida las mujeres de nuestro panel de estudio son capaces, dentro de sus áreas respectivas de trabajo, de desarrollar habilidades sustanciales y transferibles a fin de mejorar su empleabilidad y, con ella, la promoción tanto dentro de la empresa como entre empresas del sector. En segundo lugar, el método nos ha permitido mostrar la manera en que las empresas reconocen explícita e implícitamente nuevas habilidades que nacen de las TICs y en las que las mujeres participan a lo largo de su jornada laboral, y si estas habilidades exigen de un aprendizaje para el cual se facilitan cursos de formación accesibles al grupo de mujeres que forman el panel de estudio.

El estudio longitudinal se siguió durante casi todo el proyecto. Las primeras entrevistas se realizaron en el sexto mes, a partir del comienzo del proyecto de investigación, se repitieron bimensualmente hasta su finalización. Las tres primeras entrevistas fueron temáticas y las que siguieron pretendían profundizar en los objetivos arriba reseñados. Además de estas entrevistas formales, las entrevistadas seguían un diario en el que consignaban cambios en el ejercicio de sus tareas y si estos cambios estaban relacionados, ya sea directa o indirectamente, con las Tecnologías de la Información y Comunicación.

Por otro lado, desde cada país hemos realizado cuatro estudios de caso - dos para las empresas de intermediación financiera y dos para el sector del comercio minorista-, creando grupos de discusión entre las empleadas (12 mujeres para cada estudio de caso) teniendo en cuenta características del puesto de trabajo (por ejemplo, la duración de la jornada laboral y el tipo de contrato) y del centro de trabajo (como el número de empleados y el sector de actividad). El objetivo era examinar las estrategias innovadoras y el comportamiento particular de determinadas empresas respecto a las implicaciones que para su empleabilidad tienen los procesos de formación en el trabajo, así como el uso y reconocimiento de determinadas habilidades. Además, los estudios se han completado con entrevistas en profundidad a representantes sindicales, responsables de recursos humanos y formación, de los servicios informáticos y mandos medios dentro de la empresa. 


\section{LAS MUJERES MAYORES DE 45 AÑOS Y LA NUEVA ECONOMÍA}

En este apartado, nuestro interés es el de dibujar a grandes rasgos los elementos que pueden caracterizar a las mujeres empleadas en el sector de la intermediación financiera que ahora tienen entre 45 y 55 años $^{2}$. El proceso de desregularización que en los últimos diez años han experimentado todas las empresas de intermediación financiera que participan de los estudios de caso del proyecto Servemploi, se traduce en una oferta más extensiva de productos y servicios. Salvando las particularidades de cada país, hay una constante que se repite en cada una de las empresas de los estudios de caso: la reestructuración organizativa que obliga a la disminución del número de sucursales, compensada en unos casos y provocada en otros, por el traslado de las tareas administrativas al back-office.

Hemos constatado para los ocho países que forman parte del estudio que, persiguiendo el objetivo de igualar sucursales propias y equipararlas a las de los competidores, por un lado, y reducir los trámites burocráticos, por otro, las empresas de intermediación financiera se han comercializado y, consecuentemente, orientado sus servicios hacia la venta de productos. En este nuevo marco, el desarrollo y uso de la destreza y habilidades entre los empleados y empleadas para relacionarse con el cliente han ido cobrando importancia, lo que ha hecho que las cualificaciones profesionales reconocidas como tales hayan ido declinando sustancialmente en importancia para las mujeres de nuestro estudio. De hecho, podríamos argumentar que las habilidades que actualmente se requieren para trabajar en el sector financiero para aquellas personas que ocupan puestos considerados generalmente de baja cualificación son, esencialmente, las mismas habilidades requeridas para trabajar en el sector del comercio.

Es en este sentido que queremos introducir el comentario de la responsable en el área de personal de un banco alemán, para quien

[...] la comunicación entre el empleado y el cliente se ha convertido en [algo] esencial porque el trato con el cliente ha cambiado de modo radical en los últimos cinco años.

Comentario muy similar a este otro expresado por un directivo de un call-centre en Gran Bretaña:

\footnotetext{
2 El trabajo realizado en los siete países restantes que forman parte del estudio Servemploi sigue la misma metodología que presentamos para el caso español. Actualmente, los participantes en el estudio estamos trabajando en la publicación del libro The Knowledge Economy and Women's Work in European Services; concretamente, las autoras del presente artículo preparamos la redacción del capítulo «Employability for What? Skills, Training and Employee Development in European Service Work».
} 
Creo que la habilidad esencial ... es que los empleados deben centrarse en el cliente $y$, sobre todo, deben saber escuchar, así que puedan entender qué les está diciendo el cliente y no sólo creer que ya saben lo que el cliente les va a pedir.

Para cada uno de los países estudiados encontraríamos comentarios similares, confirmando la hipótesis según la cual las habilidades relacionales son las que más ampliamente se han desarrollado entre las mujeres que durante años han trabajado en tareas originariamente asignadas como administrativas dentro de las entidades bancarias. Hemos recogido que todas las empresas de intermediación financiera destinan tiempo y dinero a la formación en este campo y que han desarrollado sofisticadas aproximaciones analíticas relacionadas con habilidades para relacionarse con el cliente, entre las que destacan el trabajo en equipo y el modo más eficaz de interacción con el cliente. A su vez, este tipo de formación en la empresa tiene más aceptación entre los empleados y empleadas más jóvenes y que menos tiempo llevan trabajando en la empresa, que son, a su vez, los primeros beneficiados.

Una empleada sueca de 52 años que trabaja en un banco con 695 sucursales en todo el país ilustra con su comentario que la profesión que durante veintitrés años ha ejercido ha pasado de ser un servicio pasivo a una venta activa:

... ahora tenemos que vender más. No te puedes quedar esperando por los clientes que vienen a preguntarte. Depende de ti informarles de qué pueden tener. Tenemos que atraer al cliente... es por su bien, claro. Quiero decir que si no vienen aquí irán a otro sitio donde les den mejores condiciones.

La pregunta que surge de este comentario es la siguiente: ¿la adquisición y/o posesión de estas habilidades facilita la promoción de estas mujeres? Expresado de otro modo, ¿son los impedimentos en la adquisición de estas habilidades los que evitan la promoción de tareas «en ventanilla» a trabajos más cualificados y mejor retribuidos, consecuencia éstos de las nuevas necesidades que surgen en el actual proceso de cambio estructural?

Como nos informa una empleada de una entidad financiera danesa (42): El trabajo como cajera en un banco es justo eso: trabajo, no carrera profesional. Con esta afirmación busca explicar que hay puestos de trabajo para los que se cree que no se requieren cualificaciones específicas y que por ello no se ofrecen posibilidades de acceso a cursos de formación y, consecuentemente, se veta la promoción. Además, en Dinamarca, como en Suecia, estos trabajos están prácticamente desapareciendo, y se están anticipando al hecho de que dentro de relativamente poco tiempo el trabajo de cajera estará de más. 
En el caso de los servicios financieros en Alemania, la promoción interna es todavía importante y posible para empleados con posiciones superiores a la de cajera, a pesar de que es mucho más problemática que en el pasado. Sí que es cierto que las mujeres que están en caja y, al mismo tiempo, vendiendo productos financieros pueden promocionarse fácil y rápidamente hacia posiciones que están orientadas casi exclusivamente a los clientes, pero esta promoción está a su vez muy limitada por la ausencia de acceso a oportunidades una vez ocupados estos puestos. También están muy restringidas por los requerimientos de movilidad geográfica, lo cual limita mucho sus perspectivas de promoción a lo que está libre en el ámbito local.

Igualmente constatamos que la creciente comercialización de los bancos limita fuertemente el futuro profesional de las mujeres que han ocupado durante años posiciones administrativas en los bancos italianos, como veremos que también ocurre en los españoles. Podríamos hablar de una fuente de competencia para la promoción entre vendedores de productos financieros y empleados administrativos:

Cada vez vamos a necesitar menos personal como [empleados] administrativos y más empleados en servicios comerciales. Las mujeres que trabajan en el área comercial pueden manejarse con mayor libertad, mientras que las que se dedican a labores administrativas son consideradas como «soporte» de las primeras y sin valor profesional (Secretaria de dirección de un banco italiano).

Quizás, paradójicamente, el ambiente de los call-centers y los back-office sea buen ejemplo de lugares donde las mujeres pueden hacer carrera, justamente por tener una estructura menos jerarquizada. Hemos constatado que en los call-centers la formación de servicio al cliente es generalmente más duradera y más amplia en gama que en los puestos de trabajo dentro de las convencionales entidades financieras, cubriendo el conocimiento del producto, la capacidad técnica para llevar a cabo determinadas operaciones y habilidades relacionales. Una de las razones por las cuales la formación dentro de este ámbito es comparativamente más completa y cuidadosa se debe al hecho de que en los call-centers los empleados generalmente entran sin conocimientos previos -ya sea en servicios financieros y/o relación con el cliente- y, por ello, tienen que aprender desde el principio y sin ventajas de partida.

La formalidad en la formación de servicios financieros en los bancos por Internet es imitada por los sistemas de promoción para agentes de servicios comerciales. En un banco por Internet sueco, todos los agentes siguieron un curso de formación para promocionar su posición hasta el puesto de asesor personal del cliente, siendo de hecho una exigencia de los empleados. En el caso de Gran Bretaña, las oportunidades de promoción en los call-centers 
van del nivel más bajo hasta el puesto de líder de grupo y, posteriormente, puestos de dirección en los servicios de atención al cliente. Lo paradójico es que considerando que las iniciales condiciones salariales y horarias son comparativamente peores en las nuevas formulaciones de las empresas de intermediación financiera, todo y tener abiertas más y mejores oportunidades de promoción, las mujeres de nuestro panel de estudio se autoexcluyen.

Centrándonos en el caso español, haremos referencia explícita a cuatro ámbitos. En primer lugar, el de los estudios: las mujeres a las que nos referimos constituyen la primera generación que de manera casi generalizada pudo acceder a la educación reglada. Presentaremos el ámbito del trabajo en segundo lugar: la mayoría de estas mujeres, tras cursar estudios, tuvieron un acceso relativamente fácil al mercado laboral. A continuación, el matrimonio y la creación de una familia: el matrimonio como aspecto que pasa de ser casi obligatorio a una elección, y la familia que compite en exigencia de dedicación con el trabajo remunerado. Finalmente, dedicaremos atención especial a un cuarto estadio en la vida de estas mujeres: cuando la prejubilación aparece como perspectiva de futuro y a muy corto plazo.

Para ilustrar los diferentes apartados a los que nos hemos referido acudiremos a las respuestas recogidas del estudio longitudinal. En su mayoría son mujeres que han optado por no realizar una carrera profesional ascendente, sino, como dirían los expertos en recursos humanos, horizontal. Es justamente este tipo de carrera la que les ha permitido compatibilizar su trabajo remunerado con los roles de madre, esposa y cuidadora. Por otro lado, como mujeres con presencia en el ámbito sindical, se mueven en un entorno laboral que las ha elegido y del que se sienten representantes. Así, haciendo uso del material cualitativo de las entrevistas en profundidad, ilustraremos los apartados que siguen centrándonos en las trayectorias de vida de estas mujeres, considerándolas como un colectivo ideal para abordar los temas que tienen que ver con los cambios que la introducción de las nuevas tecnologías exige, así como con los procesos de fusión entre empresas que últimamente operan entre las grandes entidades bancarias. El mundo financiero y el de la intermediación financiera está de moda, pero lo están menos las personas que en gran medida lo constituyen.

Antes de continuar cabe una segunda puntualización: evidentemente, entre las entrevistadas se encuentran diversas pautas de relación (mujeres casadas, separadas, viudas, solteras...; con o sin hijos; viviendo con los dos padres, o con los suegros, o con alguno de ellos...). Sin embargo, tienen en común el hecho de representar una generación que ha incorporado muchos cambios que en cierta medida la sociedad le ha impuesto, ni que sea indirectamente. Así, la cuestión a la que debemos responder en primer lugar sería: ¿de quién hablamos cuando nos referimos a las mujeres mayores de 45 años? 
La primera vida adulta: estudiar, trabajar, casarse

Marina Piazza, en su libro Le ragazze di cinquant'anni (Piazza, 1999: 8), hace referencia a una generación que ahora se encuentra en pleno pasaje hacia lo que se podría llamar la segunda vida adulta. La autora las describe como mujeres nacidas entre 1950-1960, aquellas que, pensando en la España franquista, cuando llegaron los ecos de la revolución del 68 se encontraban en edades adolescentes. Son mujeres que, a diferencia de sus madres, entraron de forma masiva en el mercado de trabajo, después de conseguir unos estudios a los que sus madres no pudieron ni tan siquiera optar. Es, pues, una generación especial por los fuertes cambios sociales que ha protagonizado y protagonizará. En el presente apartado nos referiremos, entre los muchos aspectos posibles que la caracterizan, a aquellos que están relacionados con: a) la educación; b) el acceso al empleo, y c) el matrimonio y la familia.

\section{Estudiar}

Esta generación a la que nos referimos es, probablemente, la primera generación de mujeres españolas de la clase media para las que obtener un título de estudio no es la excepción, sino la regla:

Hice los estudios hasta bachillerato; la verdad es que no intenté hacer nada más. Me casé a los 20 años.

Estudié en una escuela religiosa primero y después, en una academia privada, lo que entonces se llamaba "Comercio». A los 14 años comencé a trabajar aunque seguí estudiando: bachillerato y después lo que ahora sería COU.

Puede parecer que, frente al deber impuesto de la escuela que les abría caminos negados a generaciones anteriores de mujeres, seguía imperando lo que venía a ser un modelo de «buena chica»; esto es, de estudiar no tanto para abrirse un futuro profesional, sino como un impass antes de pasar por el altar.

Evidentemente, el notable aumento de la escolarización secundaria y superior —primero para esta generación y después, de manera más generalizada, para las siguientes - fue fundamental y determinante del gran aumento en cuanto a participación femenina en el mercado laboral de los años setenta y los decenios posteriores. Paralelamente, el ingreso y, sobre todo, la permanencia en el mercado laboral vinieron estimulados por una demanda de trabajo nueva y en expansión. En particular en el sector servicios y en la Administración 
pública, que es donde se van creando puestos de trabajo, y que estimulaba una especie de círculo vicioso entre la mayor oferta femenina de empleo y la demanda de servicios.

El primer contrato fue... un contrato de cuatro meses que me prorrogaron hasta seis; luego tres meses sin trabajar y de nuevo el contrato de seis. Así estuve durante tres años, hasta que me preparé las oposiciones $y$, entonces, pues ya fija.

Yo comencé a trabajar a los 14 años hasta los 16 en un despacho, mañana y tarde, hasta que un compañero de trabajo me dijo que por qué no hacía oposiciones a un banco. Rellené un montón de solicitudes... el primer examen que hice fue para el Banco Central, aprobé y entré a trabajar. Desde el principio fui fija, era la idea de un trabajo para toda la vida por el horario y el sueldo, y de hecho yo tengo contrato indefinido desde que entré a trabajar en el banco.

Esta situación es la que permite a las mujeres seguir trabajando una vez casadas y hacer compatible el trabajo remunerado con las responsabilidades familiares. Es cierto que el sector de la intermediación financiera, por su particularidad en el horario de trabajo (tradicionalmente de 8 a 15 horas y en verano librando los sábados), facilita la permanencia de muchas de estas mujeres que han tenido que combinar su jornada laboral con la familiar. Sus trabajos les permiten, pues, una gestión de la maternidad "sostenible», aunque les haya dificultado su promoción dentro de la empresa. (En este sentido cabe añadir que, en el mismo estudio longitudinal pero con entrevistas a mujeres que trabajan o han trabajado en el sector del comercio minorista, el hecho de combinar las dos esferas se presenta como casi imposible. En este sentido, opera frecuentemente un relevo generacional o discontinuidad laboral cuando la mujer debe elegir entre seguir trabajando o formar una familia de origen —cónyuge, hijos...- y/o hacerse cargo de la familia de procedencia — padres, suegros...- . Las mujeres con cargas familiares dejan su trabajo remunerado y otras más jóvenes ocupan sus puestos. Por otro lado, algunas de estas mujeres expresan en términos de promoción abandonar sus empleos para contraer matrimonio.)

\section{Trabajar}

Hay una segunda forma de entender los cambios respecto a generaciones anteriores, quizás más fuertes, que ha afectado a muchas mujeres a las que nos venimos refiriendo. La mayoría, después de cursar estudios, encontró fácilmente y de forma rápida un trabajo en su tanteo inicial con el mercado laboral y ha sido mayoritariamente una profesión en el sector público o el financiero. Diremos, además, que no sólo fue el mercado que abrió oportunidades antes inexistentes. Cuenta, y no menos, el deseo de autonomía de 
la familia de origen, tal era también la necesidad de independencia económica - aunque medida y restringida - respecto al marido o al compañero, y era asimismo el deseo de tener una profesión que fuera poco problemática para gestionar el cuidado de los hijos y el matrimonio. Por tanto, la demanda ha correspondido perfectamente con la oferta. Muchas de estas mujeres han participado y participan voluntariamente de profesiones sin posibilidad ni perspectivas de carrera ni de grandes ganancias, quedando como estupendas mujeres juiciosas, sin madurar ambiciones y haciendo bien, incluso muy bien, el propio trabajo.

Para ilustrar este apartado hemos acudido a los comentarios de una mujer de 46 años que cursó estudios universitarios mientras trabajaba en el banco y embarazada de su primera hija:

Hace años me planteé dejar el banco y ponerme a trabajar [en la profesión en la que se había formado universitariamente]. Pero, claro, tenía una hija pequeña y mi trabajo era fijo, y sobre todo el horario de ocho a tres me permitía una serie de combinaciones con las guarderías, estar con mi hija... porque yo entonces me planteé: «dejo el banco y busco algo que es para lo que me he preparado y me gusta. Pero, entonces, ¿qué haré?, ¿atenderé problemas de otras personas y no atenderé a mi propia hija?». Entonces pensé que era incongruente. Pero ahora que mi hija no me necesita, con 45 años que tengo, a lo único que puedo aspirar es a hacer colaboraciones, de satisfacción personal, como si por las tardes fuera a una ONG.

Paralelamente, tal y como nos han relatado estas mujeres, la mayoría siente que el trabajo que realiza le otorga cierta identidad: «no podrían ser ellas mismas si les faltara el trabajo». Lo que nos interesa resaltar en este punto es lo que de cambio ha supuesto pensar de esta manera pues, para la mayoría, cuando empezó a trabajar «jamás hubiera imaginado que veinte años más tarde seguiría trabajando.» Aunque, de hecho, como tantas veces se hace en el hablar cotidiano, no suelen utilizar la palabra trabajo cuando se refieren a actividades similares no remuneradas. Así: «el empleo asalariado ha pasado a ser casi exclusivamente la única actividad designada como trabajo» (Alberdi et al., 2000: 66), aunque dediquen una gran cantidad de horas a las actividades domésticas que son también trabajo necesario para subsistir, así como a tareas administrativas que realizan para otros miembros de la familia (asesoramiento, declaraciones de renta, gestión de recibos...).

Para esta generación de mujeres que trabajan en el ámbito financiero, a diferencia de otras mujeres que también entraron en el mercado laboral, el matrimonio no ha coincidido con el retiro, momentáneo o no. Por lo tanto, no es de extrañar que sientan que el trabajo remunerado forma parte de su identidad. 
Nunca me he planteado dejar de trabajar, ni tampoco trabajar fuera del banco. Yo estoy trabajando por un cúmulo de circunstancias, pero la verdad es que nunca en la vida me imaginé que a mi edad continuaría trabajando en el banco. $O$ sea, que nunca me he planteado salir fuera del banco; de hecho, no sabría hacer otra cosa.

A mí es que me gusta el trabajo que hago. No me gusta que sea como es un tanto rutinario lo que hago, pero no me desagrada. Yo es que soy administrativa pura. Pensaba, cuando empecé a trabajar, que tendría otro aliciente, que sería de otro color... pero no me siento a disgusto, me encuentro bien porque considero que lo he escogido yo. Además, pienso que tengo que ser realista y saber que tenemos un trabajo muy por encima de la media.

Y aun siendo cierto que también a esta edad, como en tantas otras facetas de la vida, el sentido y la importancia del trabajo remunerado pueden cambiar, mayoritariamente las mujeres de las que estamos hablando han trabajado desde siempre - y así lo expresan- en un tipo de trabajo con poca responsabilidad y también pocos cambios. Es sólo recientemente que se están viendo obligadas a incorporar las transformaciones que las TICs imponen; eso o ir pensando en la prejubilación:

Yo, dentro de diez años, estaré jubilada, con 55. Y espero hacer infinidad de actividades: ir a la playa, leer, ir a museos, cantar... y hacer aquellas cosas que siempre me han gustado. Dentro de cinco estaré haciendo lo mismo, aunque, claro, ser delegada sindical es ahora una especie de seguro para mantener mi puesto de trabajo, aunque no para la promoción.

Dentro de este grupo, evidentemente, en la muestra se hace patente la ausencia de aquellas mujeres para las que este recorrido significó la oportunidad de poder aventurarse en la vida hacia la búsqueda de nuevos caminos, de la formación, pasando a las profesiones liberales, ejerciendo como «autónomas» en el doble sentido —-profesional y personal.

\section{Casarse}

Pese a que no son pocas las mujeres de esta generación que han seguido estilos de vida alternativos, casi todas las mujeres a las que hemos entrevistado se casaron a edades que ahora calificaríamos de tempranas, y casi siempre por la Iglesia. Sin embargo, en nuestro estudio no nos ha interesado centrarnos en estos aspectos. Sí lo haremos, no obstante, en aquellos otros relacionados con las relaciones de dependencia que estos contratos matrimoniales han impuesto a esta generación concreta de mujeres. Por un lado, los hijos, que 
ahora tendrían la edad en la que sus madres se emanciparon pero que no lo hacen; por otro, los padres y/o suegros, dependientes y sin un sistema de bienestar social sólido que obliga a la mayoría de estas mujeres a hacerse cargo también de ellos.

Tengo dos hijos: un chico y una chica (de 23 y 20 años), que están en casa, tranquilos y muy bien. Además, como responsabilidad tengo a mi padre, que desde que se murió mi madre — hace dos años - viene a casa a desayunar y a cenar. También tenemos un perro. Estoy muy bien, aunque mal de dinero pues me tengo que hacer cargo de todos los gastos; sólo recibo 35.000 pesetas de la pensión de viudedad, además de mi sueldo del banco; suerte que no tengo ningún gasto de hipoteca y que no tengo coche. Mi padre es joven, tiene 70 años, pero siempre vivió con mi madre, y ahora sin ella no sabe hacer nada... Él duerme en su casa, pero se pasa casi todo el día en la nuestra; me paga una señora que viene a hacer la limpieza, una vez por semana. Al mediodía, cuando yo no estoy en casa, come con los críos.

Mi hija cumplirá 18 años. Mi suegra vivía en la calle justo enfrente y supuso una carga (se murió hace un año), porque su marido había muerto hacía tiempo y pasaba mucho tiempo con nosotros, venía todos los fines de semana a casa y además le ayudaba en todas las tareas administrativas. Además, mis padres también venían los domingos, sobre todo los últimos años. Al principio todo me resultaba muy difícil: mi hija, mi suegra los fines de semana... También es verdad que, a cambio, cuando mi hija estaba enferma era ella [la suegra] quien me la cuidaba y que puntualmente nos ha ayudado a pagar algún recibo o, por ejemplo, a comprar la lavadora.

Sus vidas son una suma de vida familiar y personal, por un lado, y de vida exterior. En esta suma se combinan dificultades e incompatibilidades junto con variación y enriquecimiento. Así, podríamos interpretar que cuentan con dos aspectos complementarios: la relación con los hijos (y padres, suegros), además de las estrategias domésticas de compatibilidad del mundo familiar con el mundo exterior (de trabajo remunerado, relaciones con colegas...).

\section{La segunda vida adulta: la prejubilación o el exilio social voluntario}

De todo el material cualitativo recogido con las entrevistas en profundidad creemos que es de gran importancia considerar las perspectivas de futuro de estas mujeres, más que profundizar en las particularidades de su acceso al empleo (a lo que nos hemos referido como primera vida adulta). Así, cuando preguntamos a estas mujeres por el tipo de promoción que creen que podrían tener - tanto dentro como fuera de la empresa para la que han trabajado y están trabajando- en un plazo de cinco y diez años, respectivamente, las res- 
puestas de una gran mayoría de las entrevistadas podrían traducirse por incertidumbre. A simple vista, pues, es fácil interpretar sus respuestas en clave de desaliento; por ejemplo, nos relatan cómo dentro de sus planes para los próximos cinco o diez años entra la prejubilación, sin pensar en la posibilidad de trabajar para otra empresa o incluso montar una empresa propia como empleadas autónomas. Es como si para estas mujeres el hecho de jubilarse a una edad temprana - a los 55 años — las apartara automáticamente de la vida laboral y, si recuperamos opiniones ya reflejadas sobre lo que para ellas significa trabajar, diríamos que también les quita tanto identidad laboral como identidad personal.

Para poder describir y profundizar en la situación de estas mujeres acudiremos a la expresión de la aceptación de la lentitud (Piazza, 1999: 174). En nuestra opinión, cabe referirse de manera positiva a la fase de la vida que sigue a aquella otra en la que durante mucho tiempo el objetivo central ha consistido en tener en equilibrio precario mundos diversos, redes de significados simbólicos diversos. Efectivamente, para estas mujeres la fase de edad de entre los 24 y los 45 años ha estado llena de eventos, de toma de decisiones, de necesidades de escoger, en una especie de desorden permanente. Esta generación ha aprendido a lo largo de su primera vida adulta a combinar responsabilidad privada (el trabajo, los hijos, las tareas domésticas, en algunos casos rupturas matrimoniales) con responsabilidad laboral. Por otro lado, son mujeres para las que uno de los grandes retos ha sido el intelectual, que ha representado — como hemos ilustrado en apartados anteriores - una transgresión respecto a las generaciones precedentes de mujeres. Pero también, junto al reto, habitaba el miedo a no poder cumplirlo, de no estar a la altura de las exigencias. Todo ello hace que la vida de estas mujeres pueda verse como «constreñida a una especie de calambre permanente» (Piazza, 1999: 175).

A fin de describir este estadio hemos acudido a la figura del funámbulo que utiliza Piazza. Como subraya Paul Watzlawick (1997; citado en Piazza, 1999: 174), si observamos cómo consigue estar en equilibrio un funambulista sobre la cuerda podemos apreciar que los movimientos con los cuales tiene la pértiga no son movimientos lentos y armónicos, sino pequeños movimientos frenéticos. Justamente por ello, porque la vida que estas mujeres han soportado ha sido y todavía es convulsa, el concepto de lentitud es un concepto lejano, casi extraño. La vida estaba llena de cosas que se debían hacer, de obligaciones a las que responder. Ir despacio no era ni contemplado, ni considerado, ni consentido por el nuevo modelo social —aquel del equilibrista—, además de verse obligada a participar de cada vez más espacios: el trabajo profesional; las tareas domésticas; los hijos, padres y suegros; el sindicato... Siempre en forma, siempre veloces, dispuestas a «tomar carrerilla».

Después, este gran correr choca contra algo: puede ser que las obligaciones no se vean tan obligaciones cuando los hijos se van haciendo mayores, o quizás se deba a un senti- 
miento más interno de rebelión contra este ir deprisa, esta carrera perenne; puede ser un incidente imprevisto que colapsa la energía total del cuerpo y constriñe a la inmovilidad, «el debilitamiento del delirio de omnipotencia» (Piazza, 1999: 175), o quizás sea el proceso natural de envejecimiento.

\section{Movimientos interiores en el trabajo}

Enfrentarse a una lentitud no siempre deseada o buscada no es indoloro: la sensación que prevalece es la de sentirse de golpe inadecuada a la situación, de sentirse negada, de haber perdido irremediablemente la energía, la capacidad de esforzarse y la agilidad física y mental que era necesaria para poder estar a la altura de la pareja y/o compañeros/as de trabajo. Probablemente, más adecuado que medir el tiempo, presente o futuro, en términos de cuántas cosas se van a hacer, hasta dónde se va a llegar... provocando olas de pánico y disgusto hacia una misma, sea mejor interrogarse por el sentido y el valor que le otorgamos a los conceptos de velocidad y lentitud.

Según Piazza (1999: 177), interrogarse por el valor que otorgamos a estos dos conceptos — velocidad y lentitud - significa medirse a una misma y las propias acciones no con el metro cuantitativo, sino con el cualitativo. No es fácil, y no lo es porque, en particular para las mujeres a las que nos estamos refiriendo, el tiempo perdido en un andar lento significa tiempo que se quita a la obligación de responder a las necesidades de los otros: clientes, jefes, familiares... A tales respuestas, las mujeres, y en especial estas a las que nos referimos, han estado socializadas desde la infancia. Pero sobre todo porque el trabajo remunerado, el hacer tantas cosas y combinarlas, exige una programación del pensamiento, incluso antes de intentar hacerlas, que no consiente fácilmente la comunicación directa con los propios deseos verdaderos, que tampoco se intentan buscar, que no se consiguen.

Al principio, por tanto, la lentitud puede sólo aparecer en sus aspectos negativos, como síntoma de un envejecimiento del que se huye y que normalmente se contempla con horror. Sin embargo, si se supera esta trampa, el actuar con lentitud puede conllevar más ventajas que desventajas: a una persona lenta los demás pueden acudir con menos requerimientos; yendo lentamente se saborean mejor las cosas; uno se cansa menos y, además, se puede aprovechar mejor de lo que ve, oye, lee...

Entonces, ¿por qué no ser conscientes del elogio de la lentitud? Así lo hacen algunas mujeres de las entrevistadas: 
Dentro de diez años, con 54, espero estar en un apartamento al lado de la playa con un novio de 25 a mi lado [risas]. Dentro de cinco años espero continuar en el banco... aunque seguro que vamos a tener movida, y fuerte, porque cerrarán muchas oficinas y lo vamos a pasar mal... Yo pienso hacer lo mismo, tanto en el banco como en el sindicato, en el que llevo toda la vida, desde los 18 años...

Pero la lentitud no debería interpretarse como estancamiento, no es «no hacer». Sin embargo, observamos que estas mujeres de entre 45-55 años, con responsabilidades familiares y que llevan más de veinticinco años trabajando para la empresa, son mayoritariamente de la opinión de que no tienen ninguna posibilidad de promoción ni en la empresa para la que trabajan ni fuera. Es como si se estuvieran preparando para el anuncio de una prejubilación, y que ésta afectara no sólo a su posición laboral en la empresa, sino también fuera, y como sin ella su identidad se viera alterada.

Yo soy una administrativa pura y dura, y lo que pasa es que no me gusta vender productos... que es lo que quiere el banco.

Así, en la mayoría de los casos que hemos recogido, estas mujeres limitan su futuro profesional a la entidad financiera en la que llevan trabajando más de veinte años:

Nunca me he planteado trabajar fuera del banco. Aunque también es verdad que jamás de la vida me hubiera podido imaginar que a mi edad continuaría trabajando en el banco. O sea, que nunca me he planteado salir fuera del banco; de hecho, no sabría hacer otra cosa. Y, además, en el banco trabajas con cosas tan específicas... Tengo la base de contabilidad, pero nada más. Quizás encontraría algo como secretaria; pero, como me estoy haciendo mayor, pues ni eso...

Otro ejemplo en esta misma dirección:

Ni he intentado buscar trabajo fuera [del banco]. Yo, a mi edad [46], tendría que hacer un esfuerzo suplementario para poder encontrar fuera un trabajo bien pagado. [...], lo de la seguridad pesa mucho. A veces sí que pienso que he sido un poco cobarde, que hay gente que lo ha arriesgado y lo ha conseguido. Pero yo...

Aunque también aquí encontramos ejemplos de aceptación. En el caso que relatamos se trata de una mujer con estudios universitarios:

... Dentro de tres años, que hay elecciones, me presentaré, y si no salgo o no me presento me enviarán a una oficina, pues... imagina, con los años que llevo fuera, $y$ 
además soy una negada para vender. Claro que si hubiera una regulación de plantilla y me quedase en la calle, pues supongo que me buscaría la vida.

Que contrasta con las nuevas incorporaciones de gente joven muy formada:

... el sueldo de entrada es bajo, para ver si te adaptas o no a la cultura de la empresa. Y después puedes hacer carrera dentro de la empresa.

\section{Estrategias exteriores desde el trabajo}

Hasta aquí hemos descrito movimientos internos, fruto de una evolución ligada a una fase de la vida (la que hemos definido como segunda vida adulta), pero también fruto de un reconocimiento del ser, de toma de consciencia y de individualización de instrumentos idóneos para afrontar los cambios. Pero, sobre la base de este primer paquete de estrategias y movimientos interiores, ¿podría abrirse la posibilidad de romper con un camino de rutinas?

Actualmente, de hecho, desarrollando el mismo trabajo nos encontramos con situaciones diferentes en lo que se refiere a condiciones laborables favorables (horario y sueldos, principalmente):

Ahora en oficinas se contrata a gente de diez a una horas, pero llegan siempre un poco antes y se van después de la una. Te vienen, por tanto, en puntas de trabajo, y esto está generando mal rollo, por la presión del trabajo y porque además les tenemos que explicar cómo se hacen las cosas. Muchas oficinas de Barcelona tienen esta modalidad de part-time.

Nosotros estamos contratando gente para la oficina que vienen de empresas de trabajo temporal. Trabajan de ocho a ocho y cobran un salario mínimo; tenemos mucha gente trabajando de esta manera. También hemos empezado a trabajar con un centro que asume toda la carga administrativa de la oficina, donde las oficinas envían todo lo administrativo, para así poder dedicarnos a la venta de productos financieros.

Y al preguntar a estas mujeres si se ven capacitadas para hacer tareas de responsabilidad $y$, por tanto, menos rutinarias, la cita que hemos recogido ilustra lo que la mayoría expresan:

Yo tengo menos posibilidades de subir porque tendría que pasar por oficinas, hacer de comercial. Pero recuerdo que en la época de la fusión, cuando no había jefes 
claros, tuve que asumir un trabajo de director, tomando decisiones con relación a grandes empresas. Y lo hice. Ahora pienso que si lo hice entonces también lo podría hacer ahora, llevando un pequeño departamento. Además, pienso que tanto a nivel de organizar el trabajo y llevar un grupo de gente — quizás por todo el trabajo que llevo hecho desde el sindicato- sí que podría hacerlo.

Sin embargo, pocas de las mujeres entrevistadas han expresado que el trabajo se pueda revisar, se pueda inventar de nuevo, que pueda convertirse en una actividad más que en un trabajo verdadero y propio. Se puede «dar un salto», afrontando mayores responsabilidades y nuevos ambientes sociales, aunque no siempre equivalgan a ascensos de carrera. También se puede no querer cambiar nada formalmente, pero encontrar aquel canal en el interior del propio trabajo que permita, para el mismo cambio de actividad, hacer aquello que más apetezca, de recortar sobre uno mismo la medida del propio trabajo, y no a la inversa, de escoger, aunque en algunos casos pueda suponer una pérdida económica. También se puede querer continuar estudiando: las universidades de la tercera edad, por ejemplo, recogen este deseo. En España, sin embargo, se utiliza una imagen antigua para adaptarla a una situación nueva. Lo único que no se intenta es modular progresivamente el tránsito entre el trabajo y el período de jubilación; ni para las mujeres ni para los hombres. Es como si la prejubilación empezara ya cuando todavía se está trabajando.

Probablemente sea necesario encontrar nuevas formas de pasaje a la segunda mitad de la vida, en una sociedad que tiene a estas mujeres al margen, exiliadas, si acaso de cuidadoras invisibles, pero no parece que hagan nada para afrontar el problema, para encontrar soluciones innovadoras. Según Piazza (1999: 189), el silencio de estas mujeres en esta fase de la vida es grave, porque no asumen la fuerza de una demanda social, no apremian para crear ejemplos de buena praxis, de soluciones diversas de aquello que siempre ha sido. En este sentido, coincidimos con la autora de Le ragazze di cinquant'anni en la necesidad de que las vidas de estas mujeres puedan condensarse en un nosotras colectivo, en un nuevo sujeto social y político. También hay necesidad de que en el imaginario colectivo nazca un sujeto inédito que ahora se va construyendo, ayudado también de algunas formas institucionales, que pueda incluir a estas mujeres.

\section{INTERVENCIONES PARA LA SEGUNDA VIDA ADULTA}

En este penúltimo apartado del artículo nuestro interés se dirige hacia la valoración que las mujeres de nuestro panel de estudio longitudinal hacen de su participación en cursos de formación en sus respectivas empresas. Tal y como hemos presentado en otra publicación (Vidal y Fernández Mostaza, 2000), la política de formación de las empresas financieras no 
cumple tanto con la teoría de la compensación como con la de la acumulación: quien más nivel de conocimientos y aptitudes profesionales tiene es quien cuenta con más posibilidades de beneficiarse de la política de formación continua. Por consiguiente, la formación continua, de la forma en que se entiende y se utiliza actualmente, es fundamentalmente un mecanismo que amplía la dualidad en el mercado de trabajo, en lugar de ser un mecanismo de reducción de dicha dualidad. Estas afirmaciones quedan reflejadas por los resultados obtenidos de los estudios longitudinales y que podemos resumir en las siguientes constataciones:

La primera constatación es que la formación es considerada por los empresarios como una inversión que deben rentabilizar. Significa que las personas jóvenes con mayor nivel de estudios reglados y deseos de realizar una carrera profesional son las que más posibilidades tienen de que la empresa les ofrezca formación continua y ellas de aceptar. La segunda, que los cursos de formación se hacen muy a menudo fuera de la jornada de trabajo para aquellas personas ya empleadas en la empresa. Aquí se plantea una cuestión de importancia trascendente: no es gratis para el trabajador realizar el curso de formación; el coste es su tiempo. Es un aspecto importante sobre todo para las mujeres con cargas familiares y no identificadas con la empresa, quienes acaban escogiendo entre el tiempo de dedicación a una misma o a otros, por un lado, o realizar el curso.

Si estas mujeres rechazan sistemáticamente asistir a dichos cursos fuera de la jornada laboral están, a largo plazo, renunciando a su promoción profesional. Para poder abordar estas dos cuestiones creemos conveniente formular las siguientes preguntas: ¿quién se beneficia de la formación continua?; y ¿cuándo se hacen la mayoría de los cursos de formación, fuera o dentro de las horas de trabajo?

\section{Los beneficiarios de la formación}

Para abordar la cuestión sobre los beneficiarios de la formación continua en las empresas financieras comenzaremos por centrarnos en el sistema organizativo de la mayoría de bancos. En general, la organización de las entidades financieras parece que sigue un diseño específico para dificultar la posibilidad de una segunda vida adulta —especialmente esta segunda y larga vida adulta de las mujeres - digna, responsable y activa a las mujeres mayores de 45 años. Es frecuente encontrar entre los comentarios recogidos expresiones como «por chusco» (indicando que la categoría se ha adquirido pon antigüedad) para subrayar la situación en la que se encuentran dentro de la empresa:

Yo llevo veintiocho años trabajando en el banco. Ahora soy oficial de primera y tengo la categoría por los años que llevo. Sí que es cierto que alguna vez me han pro- 
puesto ser interventora, que es como subdelegada, pero no he aceptado porque me piden la vida y la sangre, y no estaba dispuesta. Para aceptarlo tienes que estar dispuesta a darlo todo.

... hace veintitrés años que estoy en el banco y tengo la categoría que me corresponde por los años que llevo. Desde hace diez no se hacen exámenes de promoción. Podría haber subido si me hubiera presentado a algún cargo, que normalmente te proponen... pero no lo he hecho.

En este sentido hay una gran diferencia con los jóvenes que actualmente son contratados. Como relata una de las entrevistadas para referirse a ellos:

En estos momentos, y desde hace un par de años, los nuevos que entran son universitarios, con máster, idiomas... y con unas condiciones mucho peor que las nuestras. Lo que pasa es que les dan funciones de delegado, por ejemplo, pero con un sueldo de un oficial de segunda que lleve unos trienios: le dan responsabilidad pero no le dan el sueldo que le correspondería.

Pero justamente como los determinantes de quiénes recibirán formación en la empresa están muy relacionados con los costes y rendimientos de la inversión en capital humano ocurre que, en el proceso selectivo para formar parte de esas actividades de formación, la selección de quién hará los cursos está condicionada al objetivo de asegurar que los empleados que la reciben proporcionarán para su empresa los mayores aumentos de productividad durante el mayor período de tiempo posible. Por tanto, características como la edad, el nivel de cualificación y, sobre todo, la motivación están estrechamente relacionadas con el proceso de selección para acceder a los diferentes cursos de formación:

La gente que tiene formación continua es la gente con carrera y que piensan que les pueden sacar algún partido a corto plazo.

Así, las mujeres a las que hemos entrevistado nos relatan que, a instancias de la empresa, son pocos los cursos de formación que han realizado:

... Cuanto más alto es el puesto, más a dedo va el tema de apuntarte a los cursos. Depende también de la trayectoria de la persona dentro de la oficina: a veces te pueden inducir, fomentar... que hagas esto o aquello. Pero cuando hablamos de estas personas [que sí reciben cursos de formación] estamos hablando de personas que están todo el día en la oficina y que dicen que sí a todo. 
Por otro lado, los cursos a los que sí acceden las mujeres de nuestro panel de estudio están mayoritariamente relacionados con la incorporación en sus tareas diarias de las nuevas tecnologías:

Yo entré al banco en el 71... y desde el 91 trabajo en un centro administrativo, haciendo trabajo de soporte a las oficinas, y no de cara al público... A nivel de formación, inicialmente el banco hacía cursos que duraban un mes o dos, obligatorios para todos los que entraban. También hicimos alguno para empezar con los ordenadores... pero desde la fusión los cursos son muy puntuales, para una cosa muy determinada y en un área muy concreta. Yo me apunté a un curso de algo que ya hacía, y lo aproveché para ver a compañeros y poca cosa más. Los cursos son siempre muy estructurados, para mucha gente y nunca hay plazas. Ahora, el último que he hecho — de iniciación básica al Windows - era delante de un ordenador con dos personas más: jéramos tres!

Cada año, por Intranet, nos llega un abanico de cursos para hacer y todo el mundo se tiene que apuntar como mínimo a uno o dos; otra cosa es que te llamen, o que los hagan. Normalmente son un poco rollo, cosas que ya sabemos porque las hacemos cada día.

En este sentido advertimos una sintonía muy diferente con las nuevas generaciones de mujeres que se incorporan al mercado laboral dentro del sector financiero. Para esta generación de jóvenes, lo que más se valora es la capacidad de ir incorporando constantemente maneras nuevas de hacer las mismas cosas, obligadas a estar abiertas a nuevas formas de aprender y a la necesidad de seguir formándose. Como apuntan Alberdi et al. (2000: 63):

En el mundo de la integración global de comunicaciones y de mercados, en el que se produce una rápida innovación tecnológica, el trabajo deja de ser una tarea establecida y es preciso formarse continuamente para estar a la altura de los cambios continuos. La formación como reciclaje y como imperativo de renovación tienen algo de voluntario pero también de ineludible para seguir siendo competitivas o incluso para conservar el puesto de trabajo.

¿Fuera o dentro de las horas de trabajo los cursos de formación?

Cuando el trabajo se vive como algo que da independencia económica, autonomía, posición social..., pero que también quita: de estar con la familia, de dedicar más tiempo a una 
misma, de poder elegir la maternidad..., es lógico pensar en el tiempo como fragmentado, dividido entre tiempo de estar dentro — del trabajo— y tiempo de estar fuera:

A nosotros, cada año, nos llega una hoja con la posibilidad de hacer cursillos. Yo me apunto a casi todo y luego casi nunca me llaman, o son por la tarde y por la tarde no puedo ir.

... Sí que hacen cursos de formación para vender productos, pero para campañas largas o específicas, y todo a base de muchas horas personales, de tiempo libre. Hay gente que se niega a prorrogar la jornada, pero con el relevo generacional se va a notar que la cosa cambia: no sólo porque vendrá gente mucho más preparada, sino porque también aceptarán alargar su jornada con cursos de formación.

Y es que aunque algunos cursos de formación se realizan en horas de trabajo (por ejemplo, cuando un trabajador es contratado por una entidad bancaria recibe una formación inicial antes de su incorporación a su puesto de trabajo; los días que estos trabajadores participan de los cursos de formación inicial son abonados como un salario por parte del empleador), sin embargo, muy a menudo estos cursos se hacen fuera de la jornada laboral. Aquí se plantea una cuestión de importancia trascendente: no es gratis para el/la trabajador/a realizar el curso de formación; el coste es su tiempo. Es un tema significativo sobre todo para las mujeres con cargas familiares y no identificadas con la cultura de la empresa, quienes prefieren no realizar los cursos a ceder un tiempo que consideran suyo y/o de sus familias. Si sistemáticamente estas mujeres rechazan asistir a los cursos están rechazando, implícitamente, su promoción profesional.

Por otro lado, el análisis de los estudios longitudinales permite intuir el diferente comportamiento de las mujeres con relación a su carrera profesional. Aquellas mujeres del sector financiero con edad aún joven para plantearse crear una familia, y que se dedican enteramente a su actividad y carrera profesional, son las que más se benefician de la formación formal, promoción profesional y aumento salarial. Por el contrario, aquellas mujeres que por razón de edad o de determinada composición familiar, y que optan por limitar su nivel de identificación con los objetivos de la empresa y, por consiguiente, limitar el tiempo de dedicación laboral al tiempo que exige la jornada de trabajo pactada, son las personas que pueden estar recibiendo menos formación continua como consecuencia de que las expectativas de incremento de la productividad de esta persona en el interior de la empresa son pequeñas. Si esta situación se mantiene durante años, estas personas son las que van a ocupar siempre los puestos más bajos del organigrama empresarial. Son estas mujeres las menos motivadas e identificadas con los objetivos de la empresa y, consecuentemente, esta falta de motivación e identificación con la empresa les obliga a separar el tiempo de 
fuera de la empresa del de dentro. Consecuentemente, estas mujeres tienen mayor riesgo de que sus habilidades queden obsoletas y, si están en el sector financiero, las primeras candidatas a las prejubilaciones anticipadas.

Para las mujeres más jóvenes que entran a trabajar con estudios universitarios la opción parece más clara: «Para hacer carrera te tienes que concentrar en una sola cosa», primando el interés por alcanzar los objetivos; están, pues, dispuestas a una fuerte inversión:

En la banca sabes muy bien qué tienes que hacer si quieres promocionarte: disponibilidad absoluta, ir a trabajar por las tardes, hacer todos los cursos... y ya está.

Constatando así que las desigualdades educativas entre los empleados de una misma empresa tienden a reforzar la desigualdad de oportunidades de recibir formación continua.

Finalmente, quisiéramos apuntar que, en nuestra opinión, el salto ascendente que observamos en las mujeres más jóvenes se concreta en un salto hacia una carrera más exigente y con nuevos retos, hacia la asunción de mayores responsabilidades y también de mayor poder. Son mujeres que a veces están ejercitando su propia profesionalidad en sectores tradicionalmente masculinos, y otras veces en posiciones altas, de poder. Sin embargo, este reconocimiento externo tiene dos sólidas bases en las propias mujeres: por un lado, el reconocimiento subjetivo de la gran importancia que ha tenido el trabajo en sus vidas; por el otro, el reconocimiento de la propia ambición, del querer modificar las cosas y ejercitar un poder para realizar las cosas «a su manera». Así, como nos explicó una responsable sindical de una entidad financiera:

Actualmente, la reivindicación principal de las mujeres que trabajan en la banca es la de la igualdad para la promoción. Una vez que están en esta línea, esto es incompatible con una reducción de jornada para poder conciliar vida laboral con familiar. Desde hace ya tiempo, desde el sindicato, se están haciendo campañas para justamente reducir jornadas de trabajo excesivas —tanto para hombres como para mujeres-. Porque actualmente está exageradamente ligado a la promoción la disponibilidad horaria y geográfica. El resultado es que las mujeres o bien renuncian a la vida profesional o a tener hijos.

\section{CONCLUSIONES}

A lo largo del artículo hemos querido hacer un análisis descriptivo del colectivo de trabajadoras mayores de 45 años empleadas en el sector bancario y que ocupan puestos admi- 
nistrativos. De los diferentes aspectos abordados es posible extraer algunas conclusiones sobre las características de la formación que estas mujeres reciben por parte de sus empleadores. De modo esquemático, creemos poderlas resumir como sigue:

En general, en toda la literatura que estudia el tema de la formación continua, la política de formación, en la manera como está gestionada actualmente, no es un mecanismo orientado a incrementar los niveles de empleabilidad de los trabajadores en paro o con más riesgo de engrosar las filas del desempleo o prejubilaciones. En este sentido responde más a la teoría de la acumulación que a la de la compensación: quien más nivel de conocimientos y aptitudes profesionales tiene es quien más intensamente se beneficia de la política de formación continua. Por consiguiente, la formación continua se convierte en un mecanismo que tiende a ampliar la dualidad en el mercado de trabajo en lugar de ser un mecanismo de reducción de dicha dualidad. Estas afirmaciones quedan constatadas por los resultados obtenidos de los estudios longitudinales: la formación es considerada por los empresarios como una inversión que deben rentabilizar. Significa que las personas jóvenes con mayor nivel de educación y deseos de realizar una carrera profesional son las que más pueden tener la oportunidad de que las empresas les ofrezcan formación continua y ellas de aceptar.

Los resultados de los estudios longitudinales nos han permitido comprender hasta qué punto las mujeres no motivadas por el contenido de su trabajo, en su tiempo libre, cursan carreras universitarias o participan en cursos cuyos contenidos no están directamente relacionados con su actividad laboral. Siguiendo los criterios de la literatura especializa en formación (Tuijman y Schömann, 1996), aquellos cursos que la persona realiza voluntaria e independientemente de su conexión con su actividad profesional diaria son bienes de consumo, a diferencia de los cursos de formación que están directamente relacionados con la actividad profesional diaria en el interior de la empresa y, en este supuesto, son considerados bienes de inversión. Aunque no se olvida que la división entre productos formativos para el consumo y productos formativos para la inversión no es perfecta y, al final, quizás lo más cómodo sea considerar cualquier educación como un bien de inversión.

Los cursos directamente relacionados con el incremento de las capacidades profesionales de la persona les han de permitir incrementar su nivel de productividad laboral. La financiación de estos cursos acostumbra a ir a cargo de la empresa y el trabajador pone su tiempo personal o una combinación de tiempo correspondiente a la jornada de trabajo y tiempo personal o privado

Sin embargo, los cursos de formación orientados a la promoción son considerados una inversión para la empresa que ha de rentabilizar a corto plazo. Constatamos la hipótesis de que la empresa financia principalmente cursos a aquellas personas que la empresa consi- 
dera que puede obtener una mayor rentabilidad de la inversión realizada. Como tal, son cursos que están muy direccionados a cubrir las necesidades inmediatas y concretas de capacidad profesional que necesita la empresa en cada momento y en cada uno de sus puestos de trabajo. Hoy por hoy, un plan de formación de empresa no está pensado tanto para el trabajador como para la empresa.

Como interpretación final de los resultados del trabajo cabe subrayar que, pese a la aceleración del cambio tecnológico que se está produciendo en los últimos años en el sector financiero, las empresas tienden a subestimar los beneficios de la formación justamente para aquellos trabajadores que más necesidad de ella tienen por las dificultades de adaptarse de manera autónoma a los cambios tecnológicos. Hemos podido constatar que aunque estas mujeres a las que nos venimos refiriendo expresan su opción de no querer acudir a los cursos de formación que ofrecen sus respectivas empresas fuera de su jornada laboral, es frecuente oírlas afirmar que ellas se están formando continuamente: «haciendo bien su trabajo» y que «se aprende haciendo». Estas mujeres ofrecen, fundamentalmente, la voluntad de hacer, la experiencia acumulada, los recursos amplios y disponibles a toda hora. Sin embargo, es como si la empresa y, por extensión, la sociedad demandasen de ellas una actitud que no reconocen como aptitud. Por tanto, son mujeres que cuentan con muchos recursos personales pero poquísimos recursos sociales reconocidos formalmente.

\section{BIBLIOGRAFÍA}

ALBERDI, Inés, et al. (2000): Las mujeres jóvenes en España, Barcelona: Fundación La Caixa.

BAETHGE, Martin; KITAY, Jim, y REGALIA, Ida (1999): «Managerial Strategies, Human Resource Practices, and Labour Relations in Banks: A Comparative View», en Regini et al., From Tellers to Sellers. Changing Employment Relations in Banks, Londres/Cambridge: The MIT Press, pp. 3-13.

CRESPO, Jorge, y SANZ, Ismael (2000): «La formación continua en España: Implicaciones de política económica», en Papeles de Economía Española, núm. 86, pp. 280-294.

CROMPTON, R., y BIRKELUND, G. E. (2000): «Employment and Caring in British and Norwegian banking: an exploration through individual careers», en Work, Employment and Society, vol. 14, núm. 2, pp. 331-352.

CROMPTON, R., y SANDERSON, K. (1990): Gendered Jobs and Social Change, Londres: Unwin Hyman.

DE LA TORRE PRADOS, Isabel (2000): «La dimensión social del capital humano. Formación ocupacional y formación continua», en Papeles de Economía Española, núm. 86, pp. 266-279.

LEE, D. (1982): «Beyond deskilling: skill, craft and class», en S. Wood (ed.), The Degradation of Work? Skill, deskilling and the labour process, Londres: Hutchinson.

MARTÍN ARTILES, Antonio, y ANDREU, Lope (1999): “¿Sirve la formación para tener empleo?», en Papers, núm. 58, pp. 39-73. 
MARTÍN FERNÁNDEZ, Miguel (2000): «El sector bancario ante las nuevas tecnologías», en Cuadernos de Información Económica, núm. 158, pp. 107-113

PIAZZA, Marina (1999): Le ragazze di cinquant’anni, Milán: Mondadori.

PERAITA, Carlos (2000): «Características de la formación en la empresa española», en Papeles de Economía Española, núm. 86, pp. 295-307.

REGINI, Mario (1999): «Comparing Banks in Advanced Economies: The Role of Markets, Technology, and Institutions in Employment Relations», en Regini et al., From Tellers to Sellers. Changing Employment Relations in Banks, Londres/Cambridge: The MIT Press, pp. 319-330.

- (2000): «Different Responses to Common Demands: Firms, Institutions, and Training in Europe», en European Sociological Review, vol. 13, núm. 3, pp. 267-286.

TUIJNMAN, A., y SCHÖMANN, K. (1996): «Lifelong Learning and Skill formation», en G. Schmid, J. O'Reilly y K. Schömann (eds.), International Handbook of Labour Market Policy and Evaluation, Edward Elgar, pp. 462-489.

VIDAL, Isabel, y FERNÁNDEZ MOSTAZA, Esther (2000): «Mujer y formación en el trabajo», en Management \& Empresa, núm. 25, pp. 38-39.

\section{ABSTRACT}

The aim of this study is to describe the tendencies in professional training which are currently taking place within financial sector, especially as they apply to women —aged between 45 and 55who have been working for more than twenty years in low qualification jobs. The intention has been to show that what determines who receives company training is very much related to costs and returns in human investment. Two major questions make up the background of the article: Firstly what characteristics define and distinguish the generation of women we refer to? Once the classification has been established the question is: Do these women benefit from their professional training programmes? And if not, why not? 